\title{
Are Normal Hips Being Labeled as Pathologic? A CT-based Method for Defining Normal Acetabular Coverage
}

\author{
Christopher M. Larson MD, Alexandre Moreau-Gaudry MD, PhD, \\ Bryan T. Kelly MD, J. W. Thomas Byrd MD, Jérôme Tonetti MD, PhD, \\ Stephane Lavallee PhD, Laurence Chabanas MS, Guillaume Barrier MS, \\ Asheesh Bedi MD
}

Published online: 19 November 2014

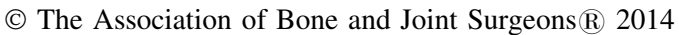

\begin{abstract}
Background Plain radiographic measures of the acetabulum may fail to accurately define coverage or pathomorphology such as impingement or dysplasia. CT scans might provide more precise measurements for overcoverage and undercoverage. However, a well-defined method for such CT-based measurements and normative data regarding CT-based acetabular coverage is lacking. Questions/purposes The purposes of the study were (1) to develop a method for evaluation of percent coverage of the femoral head by the acetabulum; and (2) to define normative data using a cohort of asymptomatic patient hip and pelvic CT scans and evaluate the variability in acetabular
\end{abstract}

One or more of the authors (LC: board membership, employment, and stock/stock options) has received funding from A3 Surgical (La Tronche, France). The institution (Grenoble University Hospital) of one or more of the authors (AM-G, JT, GB) has received funding from A3 Surgical. During the study period, an amount of less than USD 10,000 was received. One or more of the authors has received funding outside of submitted work from Smith \& Nephew (Andover, MA, USA) and A3 Surgical (CML, JWTB); Smith \& Nephew, Pivot Medical (Sunnyvale, CA, USA), and A3 Surgical (BTK); A3 Surgical (JT); outside of submitted work paid to the institution from A3 Surgical (SL); and outside of submitted work from Smith \& Nephew Endoscopy and Pivot Medical (AB). During the study period, an amount of less than USD 10,000 was received. One or more of the authors has stock/stock options in A3 Surgical (CML, JWTB, JT, SL) and A3 Surgical and Pivot (BTK). One of the authors (JWTB) had a grant paid to their institution from Smith \& Nephew. One of the authors (JWTB) receives royalties from Springer Medical (New York, NY, USA).

All ICMJE Conflict of Interest Forms for authors and Clinical Orthopaedics and Related Research ${ }^{\circledR}$ editors and board members are on file with the publication and can be viewed on request.

Each author certifies that his or her institution approved the human protocol for this investigation, that all investigations were conducted in conformity with ethical principles of research, and that informed consent for participation in the study was obtained. version for asymptomatic patients with normal lateral coverage (lateral center-edge angle [LCEA] $20^{\circ}-40^{\circ}$ ) that has previously been defined as abnormal based on radiographic parameters.

Methods Two-hundred thirty-seven patients (474 hips) with hip CT scans obtained for reasons other than hiprelated pain were evaluated. The scans were obtained from a hospital database of patients who underwent CT evaluation of abdominal trauma or pain. In addition, hips with obvious dysplasia $\left(\right.$ LCEA $<20^{\circ}$ ) or profunda $\left(\right.$ LCE $>40^{\circ}$ ) were excluded resulting in a final cohort of 222 patients (409 hips [115 men, 107 women]) with CT scans and a mean age of $25 \pm 3$ years. CT scan alignment was corrected along the horizontal and vertical axis and percent acetabular coverage

This work was performed at Grenoble University Hospital, Grenoble, France.

C. M. Larson $(\square)$

Minnesota Orthopedic Sports Medicine Institute at Twin Cities Orthopedics, 4010 West 65th Street, Edina, MN 55435, USA

e-mail: chrislarson@tcomn.com; chrislarson@ocpamn.com

A. Moreau-Gaudry, G. Barrier

Clinical Investigation Center Technological Innovation

(CIC-IT), Grenoble University Hospital, Grenoble, France

B. T. Kelly

Sports Medicine and Hip Injuries, New York-Presbyterian

Hospital/Hospital for Special Surgery, New York, NY, USA

J. W. Thomas Byrd

Nashville Sports Medicine \& Orthopaedic Center, Nashville,

TN, USA

J. Tonetti

Orthopedic Traumatology Department, Grenoble University

Hospital, Grenoble, France 
around the clockface ( 3 o'clock $=$ anterior $)$, and regional (anterior, superior, posterior) and global surface area coverage was determined. Percent coverage laterally was correlated with the LCEA and the presence and prevalence of cranial retroversion (crossover sign) and a positive posterior wall sign were determined.

Results The mean regional percent femoral head surface area coverage for the asymptomatic cohort was $40 \% \pm 2 \%$ anteriorly, $61 \% \pm 3 \%$ superiorly, and $48 \% \pm 3 \%$ posteriorly. Mean global coverage of the femoral head was $40 \% \pm 2 \%$. The local coverage anteriorly ( 3 o'clock) was $38 \% \pm 3 \%$, laterally (12 o'clock) was $67 \% \pm 2 \%$, and posteriorly (9 o'clock) was $52 \% \pm 3 \%$. The mean lateral coverage represented a mean LCEA of $31^{\circ}( \pm 1 \mathrm{SD})$. Fifteen percent of hips demonstrated cranial retroversion that would correlate with a crossover sign, and $30 \%$ had $<50 \%$ posterior coverage that would correlate with a positive posterior wall sign on an anteroposterior pelvis radiograph. In addition, male hips had a higher prevalence of a crossover sign (19\%; $95 \%$ confidence interval $[\mathrm{CI}], 14 \%-25 \%$ versus $11 \%$; $95 \% \mathrm{CI}, 7 \%-16 \%$; $\mathrm{p}=0.03)$ and posterior wall sign (46\%; 95\% CI. 39\%-53\% versus 13\%; 95\% CI, 9\%-19\%; $\mathrm{p}<0.001)$ compared with women. A positive crossover sign or posterior wall sign was present for 113 male hips $(53 \%$; $95 \%$ CI, 46\%-60\%) compared with 39 female hips (20\%; 95\% CI, 15\%-26\%; p < 0.001$)$.

Conclusions This study provides normative coverage data and a reproducible method for evaluating acetabular coverage. Cranial acetabular retroversion (crossover sign) and a positive posterior wall sign were frequent findings in a young asymptomatic cohort and might be a normal variant rather than pathologic in a significant number of cases. Level of Evidence Level III, diagnostic study.

\section{Introduction}

Femoroacetabular impingement (FAI) and hip dysplasia typically are diagnosed based on plain radiographic parameters such as the lateral center-edge angle (LCEA), anterior center-edge angle (ACEA), crossover sign, and posterior wall sign. None of these parameters in isolation, however, may be sufficiently sensitive to identify and differentiate dysplastic or impingement morphologies in all cases and these diagnostic challenges have been previously reported [4, 9, 17, 27]. Acetabular retroversion, for

S. Lavallee, L. Chabanas

A3 Surgical, La Tronche, France

A. Bedi

MedSport, Department of Orthopaedic Surgery, University of Michigan, Ann Arbor, MI, USA example, can be seen in the setting of dysplasia as well as FAI, and whether acetabular retroversion represents a truly pathologic condition in all cases is unclear [29]. CT scans and three-dimensional (3-D) imaging might provide more accurate estimates with regard to these factors; however, the optimal method for using CT imaging in this setting has not been well defined. In addition, we lack normative data with regard to acetabular coverage of the femoral head in asymptomatic individuals based on CT scans. It may be critical to have a predictable and accurate method for evaluating normal and abnormal acetabular coverage when considering management for hip disorders in an effort to optimize surgical results and minimize the potential for complications. Iatrogenic instability or residual impingement can result from surgical treatment based on an inaccurate diagnosis secondary to this lack of data regarding the normal variability in hip morphology in asymptomatic patients.

The purpose of the current study was to develop a novel method for defining acetabular morphology based on percent acetabular coverage of the femoral head at every clockface location. The second purpose of the study was to use this method to define normal parameters of acetabular coverage using a cohort of asymptomatic patient hip and pelvis CT scans in an effort to define the normal variability in acetabular morphology that might have been previously regarded as pathologic.

\section{Patients and Methods}

This study was approved by the institutional review board from Grenoble Hospital, Grenoble, France. A deidentified database of CT scans of the abdomen and pelvis obtained for reasons other than symptomatic hip disease was used. The current cohort was obtained from patients seen in the emergency department at Grenoble Hospital, Grenoble, France, with CT scans obtained for further evaluation of atraumatic and traumatic abdominal pain. CT scans of asymptomatic patients aged 20 to 35 years of age were eligible for inclusion in the study, and those with evidence of Tönnis Grade I or greater degenerative changes of the hip, previous hip surgery, or hip fracture were excluded from the study population. A total of 274 studies were selected based on these criteria, but $14 \%(\mathrm{n}=37)$ were excluded for technical issues, including an incomplete examination $(\mathrm{N}=31)$, image series spacing $>2.5 \mathrm{~mm}$ $(\mathrm{N}=3)$, or metal artifacts $(\mathrm{N}=3)$. A total of 237 patients (119 men, 118 women [474 hips]) were therefore available with a mean age of 25 years (men, $25 \pm 3$ years, women 25 years \pm 4 years). The specific scans included were CT angiogram-abdomen/pelvis $(\mathrm{n}=21)$, thoracoabdominal aortic CT $(\mathrm{n}=1)$, abdominal CT $(\mathrm{n}=63)$, abdominal 
pelvis CT ( $\mathrm{n}=146)$, CT angiogram-pelvis lower extremity $(\mathrm{n}=3)$, and neck thoracoabdominal CT $(\mathrm{n}=3)$.

To exclude occult cases of significant acetabular undercoverage (dysplasia) or global overcoverage (profunda and/or protrusio) from the analysis, cases with a LCEA of $<20^{\circ}(2 \%$ [n $=8$ hips $)$ or $>40^{\circ}(12 \%$ [n $=57$ hips $\left.]\right)$ in the frontal plane were identified (total, $\mathrm{n}=65$ hips, 50 patients) and excluded from the analysis. Of the 65 excluded hips, 30 were bilateral exclusions (15 patients) and 35 hips were unilateral exclusions (35 patients) in 21 men and 29 women. The remaining 222 patients (115 men [213 hips] and 107 women [196 hips] [409 total hips]) were included in the final analysis. More women were excluded from the initial cohort, which was not surprising because acetabular dysplasia and global acetabular overcoverage (both of which were excluded) has been shown to be more common in women. However, there remained no difference between the proportion of female and male hips before and after exclusion (proportion of women hips before exclusion 50\%; $95 \%$ confidence limit [CI], $43 \%-56 \%$ and after exclusion $48 \%$; $95 \%$ CI, $41 \%-55 \% ; \mathrm{p}=0.8)$.

\section{Computer-based Analysis Three-dimensional Coverage} Measurement

The software used in this study was developed by the authors to compute anatomic landmarks and to characterize the acetabulum in 3-D CT scan images. A bilateral pelvis 3-D CT scan from the anteroinferior iliac spines to a minimum of $1 \mathrm{~cm}$ below the lesser trochanter is used.

A best-fit sphere is calculated to determine the femoral head on each side. Reference axes are then standardized to avoid confounding from variable patient positioning in the CT gantry. The image coordinate system is realigned to the pelvic coordinate system defined by the line joining the femoral head centers to correct for axial rotation and frontal plane rotation of the pelvis. Pelvic tilt is also corrected by aligning the frontal plane with the anterior pelvic plane (APP) as [19] defined by the anterosuperior iliac spines and the pubic symphysis.

The acetabular volume is separated from the femoral head volume and a 3-D hemipelvis bone volumetric model of the side of interest is constructed. From each hemipelvis model, the acetabular rim is detected and generates a series of points distributed radially every $7.5^{\circ}$ along the rim.

A clockface reference is constructed such that the 6 o'clock position corresponds to the most inferior point of the acetabular notch. The 12 o'clock position is then defined as opposite the 6 o'clock position relative to the radial axis. The clockface reference system along with the acetabular axis enables the computation of a radial rim curve describing the local shape of the rim at any clock position in a manner completely independent from the global acetabular orientation and pelvic tilt.

Reformatted radial slices are reconstructed around the horizontal axis for all clock positions. In each radial slice a local coverage percentage $(\mathrm{C} \%)$ is computed as the circumferential portion of acetabular roof from the horizontal axis to the acetabular rim border point included in this slice $\mathrm{C} \%=\theta / 180^{\circ} \times 100$.

Acetabular rim border points are further projected radially on the best-fit sphere approximating the femoral head. Global surface coverage percentages are then computed in three directions: anterior, superior and posterior. A global 3-D coverage percentage is also computed from the projection of the acetabular rim curve onto the best-fit sphere of the femoral head. The portion of the sphere surface, which is covered by the projected curve relative to the full surface of the sphere, determines a 3-D surface coverage percentage. The mean global percentage coverage was subsequently separated for men versus women to identify any differences in sex.

\section{Correlation With Conventional Radiographic Parameters}

\section{Lateral Center-edge Angle}

Reformatted vertical slices are reconstructed around the realigned vertical axis passing through the femoral head center from posterior to anterior positions. In each vertical slice, the local acetabular center-edge angle is computed as the angle between the vertical axis and a line joining the femoral head center to the border point of the rim included in this slice. The standard LCEA is defined from the vertical slice for which the rim border point is most lateral. This 3-D definition ensures that the center-edge angle is always computed on a rim border point and not on an artifact resulting from radiographic projections.

\section{Crossover Sign}

Jamali et al. [16] validated the use of the crossover sign to detect cranial acetabular retroversion on AP pelvic radiographs. The reliability of the $\mathrm{C}$ crossover sign OS, however, is predicated on a well-positioned radiograph with standardized tilt and obliquity. To identify cases of focal retroversion that would correlate with a true crossover sign on a plain AP radiograph, the incidence of retroversion based on percentage coverage data was 
determined at 1 o'clock, 2 o'clock, and 3 o'clock positions. Those cases with a change in relative anterior to posterior coverage between clockface locations were recorded as a positive crossover sign.

\section{Posterior Wall Sign}

The posterior wall sign was described by Reynolds et al. [26] and defined the coverage of the femoral head by the posterior wall in relation to the center of the femoral head on a well-positioned, AP pelvic radiograph. A posterior wall that is medial to the head center at the 9 o'clock position was reported to be abnormal in this study and defined as positive for possible posterior wall insufficiency and/or global retroversion. To correlate the posterior 3-D coverage data in the current study with a true radiographic posterior wall sign, the incidence of posterior femoral head coverage $<50 \%$ at 9 o' clock was determined and recorded.

\section{Statistical Analysis}

The analysis described was applied to the 409 asymptomatic hip cases, generating the measurements described for each case. For each measurement, the average and SD were computed.

Quantitative parameters are described by their average and SD. Qualitative parameters are described in numbers and percentage.

For the proportion comparison, the chi square test is implemented to compare proportions. In the event the conditions of the test are not met (insufficient numbers), Fisher's test is used.

For the average comparison, Student's t-test is implemented to compare quantitative variables. If the conditions of the test are not met, a nonparametric test is used. If normality is demonstrated but not variance equality, the Welch test is used; if normality is not demonstrated, the Mann-Whitney test is used.

To evaluate correlation between two quantitative parameters, the Pearson coefficient of correlation is computed. A correlation test and a linear regression are also computed.

All statistical tests were performed using $\mathrm{R}$ software (version superior to 2.15; R Development Core Team, Vienna, Austria).

\section{Results}

The mean percent regional femoral head surface area coverage for the asymptomatic cohort was $40 \% \pm 2 \%$

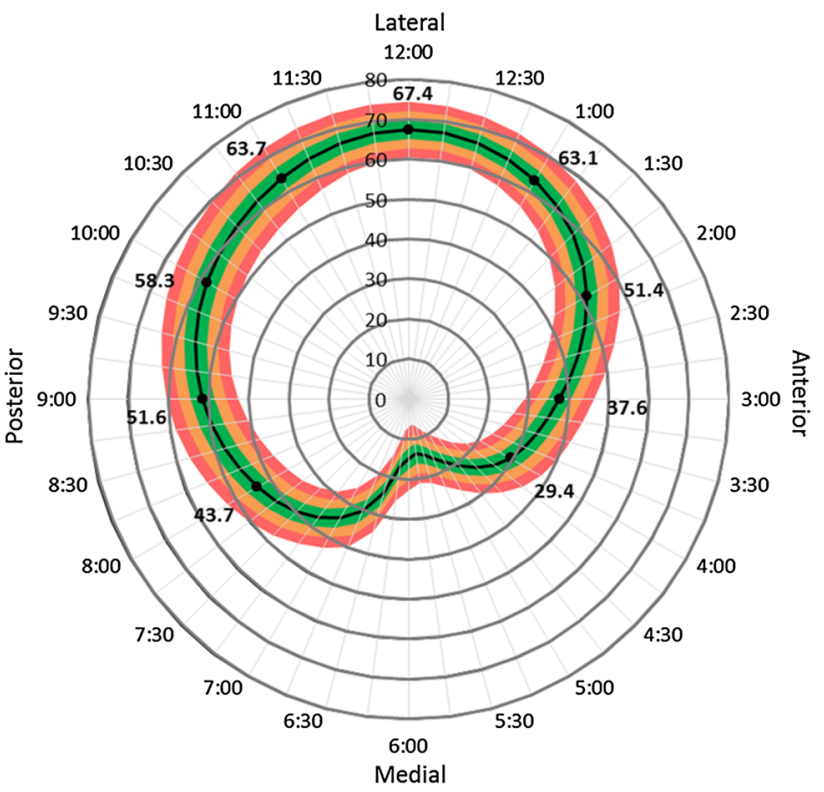

Fig. 1 The surface area coverage percentages were computed from the projection of the acetabulum rim curve onto the best-fitting sphere of the femoral head. The mean coverage percentage \pm 3 SD at each clockface location is shown: Red line $= \pm 3 \mathrm{SD}$; orange line $= \pm 2$ $\mathrm{SD}$; green line $= \pm \mathrm{SD}$; black line $=$ average .

anteriorly, $61 \% \pm 3 \%$ superiorly, and $48 \% \pm 3 \%$ posteriorly. The mean local coverage anteriorly ( 3 o'clock) was $38 \% \pm 3 \%$, laterally (12 o'clock) was $67 \% \pm 2 \%$, and posteriorly (9 o'clock) was $52 \% \pm 3 \%$ (Fig. 1). Locally, no difference was identified at 12 o'clock $(67 \% \pm 2 \%$ in men versus $67 \% \pm 2 \%$ in women; CI of difference of means, -0.6934428 to $0.2687278 ; \mathrm{p}=0.38$ ), but differences were identified at 3 o'clock $(39 \% \pm 2 \%$ in men versus $36 \% \pm 3 \%$ in women; CI of difference of means, -3.031622 to $-2.006859 ; \mathrm{p}<0.001)$ and 9 o'clock $(50 \% \pm 3 \%$ in men versus $53 \% \pm 3 \%$ in women; CI of difference of means, 1.965739-3.021774; $\mathrm{p}<0.001)$. At all clockface locations men had more relative retroversion compared with women (Fig. 2). Mean global percent coverage of the femoral head was $40 \% \pm 2 \%$. No difference was identified between men and women for mean global percentage coverage $(40 \% \pm 2 \%$ [men], versus $40 \% \pm 2 \%$ [women]; CI of difference of means, -0.3156769 to $0.6031541 ; \mathrm{p}=0.54)$.

\section{Correlation With Conventional Radiographic Parameters}

The mean lateral coverage (12 o'clock) in the cohort was $67 \% \pm 2 \%$, which correlates with a LCEA of $31^{\circ}$ with a range $27^{\circ}$ to $36^{\circ}$ (Pearson's product-moment correlation = 0.98; Fig. 3). 


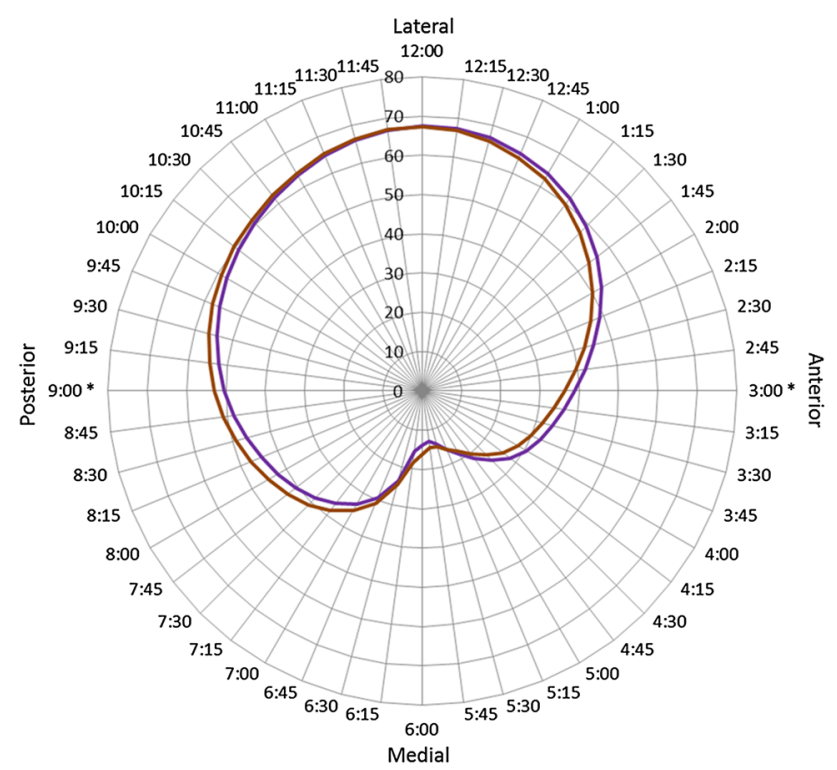

Fig. 2 Differences in local coverage between men (purple line) and women (brown line) is demonstrated. There was no statistically significant difference identified at 12 o'clock $(\mathrm{p}=0.38)$. However, statistically significant differences were identified at 3 o'clock and 9 o'clock $(\mathrm{p}<2.2 \mathrm{e}-16)$. At any clockface location, men had more relative retroversion compared with women.

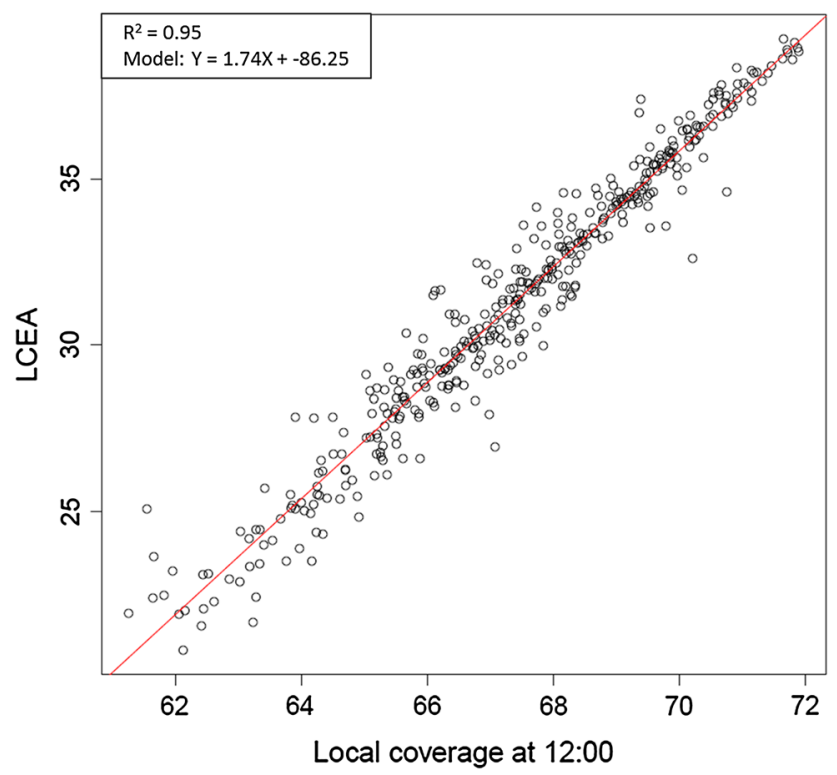

Fig. 3 The correlation between local acetabular coverage at 12 o'clock and the LCEA is shown. The Pearson's correlation coefficient was 0.98 for this regression model.

The presence of cranial retroversion, indicated by using the crossover sign, was present in $15 \%$ of all hips. Men demonstrated a greater prevalence of cranial retroversion than women $(19 \%$; $95 \%$ CI, $14 \%-25 \%$ versus $11 \%$; $95 \%$ CI, 7\%-16\%) of hips, respectively; Table $1 ; \mathrm{p}=0.03$;
Table 1. Presence of a radiographic posterior wall sign using CTbased analysis of femoral head coverage

\begin{tabular}{llll}
\hline Posterior wall percentage & All hips & Male & Female \\
\hline$<50 \%$ & $30 \%$ & $46 \%$ & $13 \%$ \\
$\geq 50 \%$ & $70 \%$ & $54 \%$ & $87 \%$ \\
\hline
\end{tabular}

Table 2. Presence of a radiographic crossover sign using CT-based analysis of acetabular version

\begin{tabular}{lllll}
\hline \multirow{5}{*}{} & \multicolumn{4}{l}{ Crossover sign $\left(\right.$ version value $\left.<0^{\circ}\right)$} \\
\cline { 2 - 5 } & Number of hips & At 1 o'clock & At 2 o'clock & At 3 o'clock \\
\hline All hips & 409 & $15 \%$ & $1 \%$ & $0.0 \%$ \\
Male & 213 & $19 \%$ & $0.9 \%$ & $0.0 \%$ \\
Female & 196 & $11 \%$ & $2 \%$ & $0.0 \%$ \\
\hline
\end{tabular}

Table 2$)$. A positive posterior wall sign $(<50 \%$ coverage at 9 o'clock) was present in $30 \%$ of cases and occurred more frequently in men than women $(46 \%$; $95 \%$ CI, 39\%-53\% [men] versus 13\%; 95\% CI, 9\%-19\% [women]; $\mathrm{p}<0.001$; Fig. 4). Overall, 152 cases (37\%; 95\% CI, 32\%-42\%) had either a positive crossover sign or posterior wall sign in the current cohort. A positive crossover sign or posterior wall sign was present for 113 male hips $(53 \%$; 95\% CI, 46\%$60 \%)$ compared with 39 female hips (20\%; 95\% CI, $15 \%$ $26 \% ; \mathrm{p}<0.001)$.

\section{Discussion}

Prior studies have primarily used plain radiographic parameters to define the acetabulum as dysplastic, normal, or impingement morphology [4, 7, 16, 20, 24, 27, 28, 30]. These radiographic parameters are unfortunately limited, because they assess only a focal region of the acetabular rim (LCEA, ACEA), the relationship of one portion of the rim relative to another (crossover sign), or are listed as present or absent (crossover sign, posterior wall sign). Therefore, they may fail to identify and quantify the severity of acetabular deformity, and their reliability to identify dysplasia or impingement morphology even among experienced hip surgeons is reported to be poor [4]. We therefore used CT scans from asymptomatic subjects to define normal acetabular morphology and coverage of the femoral head. We found that cranial retroversion as assessed with the crossover sign and the posterior wall sign were frequent findings in a young asymptomatic cohort.

Limitations of the current study include defining normative data for acetabular coverage based on a young, asymptomatic population. Studies have shown that a substantial proportion of asymptomatic patients have radiographic evidence of hip 


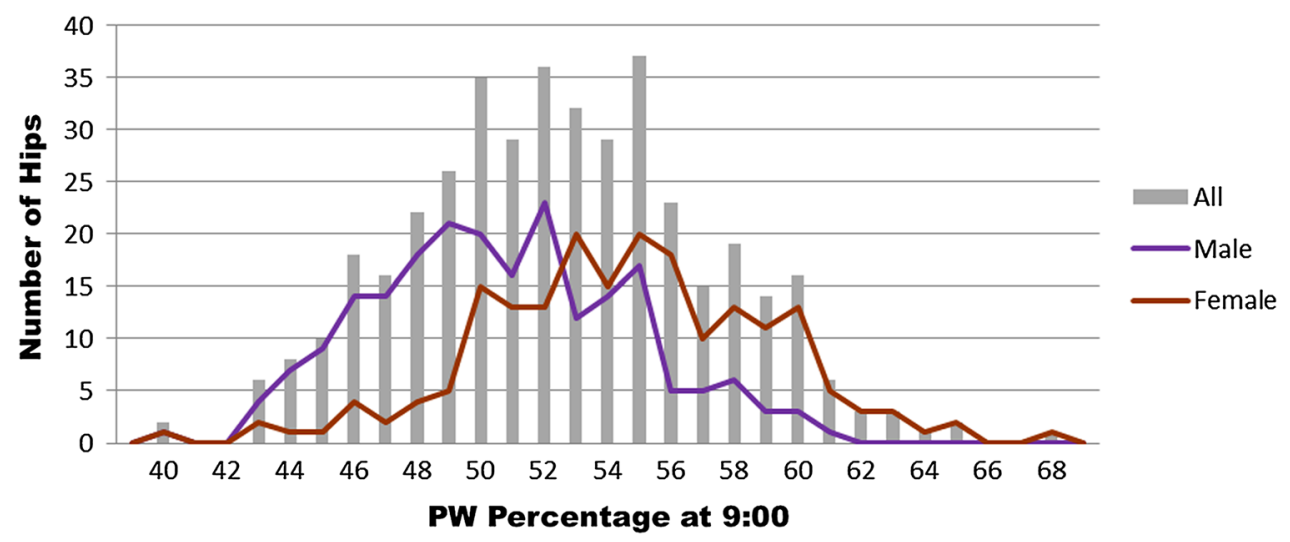

Fig. 4 Distribution of posterior femoral head coverage based on local percent coverage at 9 o'clock is represented. A posterior wall (PW) sign, or $<50 \%$ coverage at 9 o'clock, was present in $30 \%$ of cases in this series.

deformity depending on activity level and the specific population studied $[8,10,12,17,18]$. We excluded hips with a LCEA $<20^{\circ}$ and $>40^{\circ}$ in an attempt to minimize the inclusion of clearly dysplastic and globally overcovered hips in the analysis. One potential concern is that these patients were not specifically evaluated with regard to hip-related symptoms and therefore might not represent a truly asymptomatic patient population. The CT scans, however, were obtained for nonhip-related symptoms, and the authors believe that this cohort still holds value as a representative sample of normal hip morphology in patients not presenting for hip-related complaints. Prior studies have shown that women have more acetabular anteversion and a greater prevalence of global acetabular overcoverage compared with men $[2,13,14,18]$. Excluding those patients with a LCEA $>40^{\circ}$ to have a cohort that was both symptom-free and without obvious global overcoverage (profunda), which is typically seen in women, may have created a more homogeneous population with less sex-based variability. Our aim, however, was to exclude patients with those morphologies (global overcoverage/dysplasia) that have been shown to have a greater risk for the development of osteoarthritis and symptoms. The LCEA, crossover sign, and posterior wall sign were calculated using CT imaging. The prevalence based on CT measurements might not be completely representative of plain radiographic measurements. However, prior studies have supported that $\mathrm{CT}$ and plain radiographic LCEA measurements have a high correlation $[12,15,20]$ and retroversion based on plain radiographs may be an overestimate of retroversion based on CT measures [30]. Therefore, the prevalence of crossover and posterior wall signs in our study might underestimate the actual prevalence in comparison to measurements based on plain radiographs. A number of pincer-type (global retroversion and focal anterior overcoverage) and dysplastic (excessive acetabular anteversion) variants, however, might not be excluded using these exclusionary criteria in the current study. Although the presence of an aspherical femoral headneck junction (cam-type FAI) could potentially affect percent coverage analysis if the area of femoral asphericity was included, we used the best-fit sphere to perform these calculations and therefore eliminate this confounding variable. The model also does not specifically assess the coverage of the load-bearing articular cartilage of the best-fit sphere and in this regard does not reflect the potential impact of variable femoral version measures and does not account for variability in the size of the lunate fossa, a region devoid of articular cartilage. The current study used a novel method for evaluating percentage acetabular coverage of the femoral head around the acetabular rim using CT scans from a young, asymptomatic population. This allowed for an accurate assessment of acetabular coverage at multiple points around the acetabular rim as well as a calculation of regional (anterior, lateral, posterior) and global surface area coverage. Previous studies have attempted to define CT-based and/or 3D morphology of the acetabulum $[1,3,5,6,11,12,15,21-23$, 25]. None of these studies to date, however, have provided a true 3-D assessment of percentage surface area coverage of the femoral head that accounts for pelvis orientation or further correlated these findings with clinically relevant radiographic parameters of acetabular deformity. Future studies might evaluate both the femoral and acetabular sides to better define true load-bearing articular cartilage coverage. Percent coverage alone does not account for all variables with respect to acetabular morphology. Acetabular inclination, for instance, plays a major role in hip mechanics.

The current CT-based study defined normative acetabular coverage measurements in asymptomatic patients and found that cranial retroversion as indicated by the crossover sign and posterior wall sign was common in asymptomatic patients. Although commonly used as a basis for diagnosis and surgical treatment, a number of plain radiographic acetabular parameters has been shown to have 
limited reliability and accuracy for defining acetabular morphology. Wassilew et al. [28] evaluated plain radiographs with regard to the crossover sign and posterior wall sign compared with 3-D CT scans relative to the true osseous APP. The positive predictive value for identifying a positive crossover sign was $36 \%$ and for identifying a positive posterior wall sign was $35 \%$ on plain radiographs compared with the CT APP reference [28]. Zaltz et al. [30] reported that 38 of $41(93 \%)$ AP plain radiographs had a positive crossover sign but only 19 of those 38 hips had focal or true acetabular retroversion on 3-D CT analysis. In addition, Clohisy et al. [4] reported a study of six highvolume hip surgeons who performed a blinded review of 25 control hips, 25 hips with dysplasia, and 27 hips with FAI. Investigators measured acetabular version, inclination, femoral head position, sphericity, and offset and categorized each hip as normal, dysplastic, or FAI on two separate occasions [4]. The majority of radiographic parameters had poor combined intraobserver $(\kappa$ range, $0.30-0.76)$ and interobserver ( $\kappa$ range, 0.19-0.64) reliability [4]. The interobserver reliability for making a diagnosis was also subjectively poor indicating that both individual radiographic parameters and a combination of the various radiographic parameters had poor reliability for diagnosing hip pathomorphology between clinicians [4]. These past studies reporting poor reliability for defining hip pathomorphology with plain radiographs and improved reliability and accuracy with 3-D CT scans should give the surgeon serious pause with regard to defining hip disorders and anatomy based on plain radiographs alone. Of greater interest is the high prevalence of cranial retroversion, crossover sign, and posterior wall sign in this asymptomatic cohort. This seriously calls into question making the diagnosis of acetabular pathomorphology or pincer-type FAI based on these plain radiographic and CT parameters. It is possible that surgeons are not only overdiagnosing these disorders, but, of even greater concern, performing surgery for normal anatomic variants. The current authors have become much more conservative with surgical resections on the acetabulum in the presence of cranial retroversion based on these findings. Future studies are required to evaluate the natural history of patients with varying degrees of acetabular retroversion and potentially redefining acetabular disorders based on the development of symptoms and potentially using regional and global acetabular coverage data rather than plain radiographic parameters. These studies would allow us to define whether these findings represent pathologic undercoverage or overcoverage versus simply normal variants.

The current results provide the clinician with a more accurate and reliable means of evaluating a particular patient's acetabular morphology around the clockface and provide normative data to define them as undercovered, overcovered, or within the normal range. Using this novel CT-based technique of analysis, we found the mean percent femoral head surface area coverage for the asymptomatic cohort was $40 \% \pm 2 \%$ anteriorly, $61 \% \pm 3 \%$ superiorly, and $48 \% \pm 3 \%$ posteriorly. Mean global coverage of the femoral head was $40 \% \pm 2 \%$. The local coverage anteriorly (3 o'clock) was $38 \% \pm 3 \%$, laterally (12 o'clock) was $67 \% \pm 2 \%$, and posteriorly ( 9 o'clock) was $52 \% \pm 3 \%$. The current study also reports a high prevalence of the crossover sign and/or posterior wall sign in asymptomatic hips (37\%) indicating that these are not necessarily pathognomonic for "pincer-type" impingement and/or posterior instability. This raises the question as to whether a large proportion of these hips is destined for trouble or simply normal variants. Based on the current findings, the variability reported for acetabular morphologies and coverage requires further study to better define these important questions.

Acknowledgments We thank Professor Ivan Bricault $\mathrm{MD}, \mathrm{PhD}$, Radiology Department, Grenoble University Hospital, Grenoble, France, for his advice and help on the data collection process.

\section{References}

1. Anda S, Svenningsen S, Dale LG, Benum P. The acetabular sector angle of the adult hip determined by computed tomography. Acta Radiol Diagn (Stockh). 1986;27:443-447.

2. Atkinson HD, Johal KS, Willis-Owen C, Zadow S, Oakeshott RD. Differences in hip morphology between the sexes in patients undergoing hip resurfacing. J Orthop Surg Res. 2010;5:76.

3. Buller LT, Rosneck J, Monaco FM, Butler R, Smith T, Barsoum WK. Relationship between proximal femoral and acetabular alignment in normal hip joints using 3-dimensional computed tomography. Am J Sports Med. 2012;40:367-375.

4. Clohisy JC, Carlisle JC, Trousdale R, Kim YJ, Beaule PE, Morgan P, Steger-May K, Schoenecker PL, Millis M. Radiographic evaluation of the hip has limited reliability. Clin Orthop Relat Res. 2009;467:666-675.

5. Cobb J, Logishetty K, Davda K, Iranpour F. Cam and pincer impingement are distinct, not mixed: the acetabular pathomorphology of femoroacetabular impingement. Clin Orthop Relat Res. 2010;468:2143-2151.

6. Dandachli W, Kannan V, Richards R, Shah Z, Hall-Craggs M, Witt $\mathrm{J}$. Analysis of cover of the femoral head in normal and dysplastic hips: new CT-based technique. J Bone Joint Surg Br. 2008:90:1428-1434.

7. Dandachli W, UI Islam S, Richards R, Hall-Craggs M, Witt J. The influence of pelvic tilt on acetabular orientation and cover: a three-dimensional computerised tomography analysis. Hip Int. 2013:23:87-92.

8. Gerhardt MB, Romero AA, Silvers HJ, Harris DJ, Watanabe D, Mandelbaum BR. The prevalence of radiographic hip abnormalities in elite soccer players. Am J Sports Med. 2012;40: 584-588.

9. Gosvig KK, Jacobsen S, Sonne-Holm S, Palm H, Troelsen A. Prevalence of malformations of the hip joint and their relationship to sex, groin pain, and risk of osteoarthritis: a populationbased survey. J Bone Joint Surg Am. 2010;92:1162-1169. 
10. Hack K, Di Primio G, Rakhra K, Beaule PE. Prevalence of cam type femoroacetabular impingement morphology in asymptomatic volunteers. J Bone Joint Surg Am. 2010;92:2436-2444.

11. Haimerl M, Schubert M, Wegner M, Kling S. Anatomical relationships of human pelvises and their application to registration techniques. Comput Aided Surg. 2012;17:232-239.

12. Hansen BJ, Harris MD, Anderson LA, Peters CL, Weiss JA, Anderson AE. Correlation between radiographic measures of acetabular morphology with 3D femoral head coverage in patients with acetabular retroversion. Acta Orthop. 2012;83: 233-239.

13. Hetsroni I, Dela Torre K, Duke G, Lyman S, Kelly BT. Sex differences of hip morphology in young adults with hip pain and labral tears. Arthroscopy. 2013;29:54-63.

14. Hildebrand F, Shin HO, Flötotto L, Krettek C, Gerich TZ. The prevalence of reduced acetabular anteversion in asymptomatic patients: a retrospective analysis. Z Orthop Unfall. 2012;150: 601-606.

15. Humbert L, Carlioz H, Baudoin A, Skalli W, Mitton D. 3D evaluation of the acetabular coverage assessed by biplanar X-rays or single anteroposterior X-ray compared with CT-scan. Comput Methods Biomech Biomed Eng. 2008;11:257-262.

16. Jamali AA, Mladenov K, Meyer DC, Martinez A, Beck M, Ganz R, Leunig M. Anteroposterior pelvic radiographs to assess acetabular retroversion: high validity of the 'cross-over-sign.' $J$ Orthop Res. 2007;25:758-765.

17. Kang ACL, Gooding AJ, Coates MH, et al. Computed tomography assessment of hip joints in asymptomatic individuals in relation to femoroacetabular impingement. Am J Sports Med. 2010;38:1160-1165.

18. Larson CM, Sikka RS, Sardelli MC, Byrd JW, Kelly BT, Jain RK, Giveans MR. Increasing alpha angle is predictive of athleticrelated 'hip' and 'groin' pain in collegiate National Football League prospects. Arthroscopy. 2013;29:405-410.

19. Lewinnek GE, Lewis JL, Tarr R, Compere CL, Zimmerman JR. Dislocations after total hip-replacement arthroplasties. J Bone Joint Surg Am. 1978;60:217-220.
20. Monazzam S, Bomar JD, Cidambi K, Kruk P, Hosalkar H. Lateral center-edge angle on conventional radiography and computed tomography. Clin Orthop Relat Res. 2013;471:22332237.

21. Murphy SB, Kijewski PK, Millis MB, Harless A. Acetabular dysplasia in the adolescent and young adult. Clin Orthop Relat Res. 1990;261:214-223.

22. Murphy SB, Kijewski PK, Simon SR, Chandler HP, Griffin PP, Reilly DT, Penenberg BL, Landy MM. Computer-aided simulation, analysis, and design in orthopedic surgery. Orthop Clin North Am. 1986;17:637-649.

23. Nakahara I, Takao M, Sakai T, Nishii T, Yoshikawa H, Sugano $\mathrm{N}$. Gender differences in 3D morphology and bony impingement of human hips. J Orthop Res. 2011;29:333-339.

24. Nepple JJ, Martel JM, Kim YJ, Zaltz I, Clohisy JC; ANCHOR Study Group. Do plain radiographs correlate with CT for imaging of cam-type femoroacetabular impingement? Clin Orthop Relat Res. 2012;470:3313-3320.

25. Philippot R, Wegrzyn J, Farizon F, Fessy MH. Pelvic balance in sagittal and Lewinnek reference planes in the standing, supine and sitting positions. Orthop Traumatol Surg Res. 2009;95:70-76.

26. Reynolds D, Lucas J, Klaue K. Retroversion of the acetabulum. A cause of hip pain. J Bone Joint Surg Br. 1999;81:281-288.

27. Siebenrock KA, Kalbermatten DF, Ganz R. Effect of pelvic tilt on acetabular retroversion: a study of pelves from cadavers. Clin Orthop Relat Res. 2003;407:241-248.

28. Wassilew GI, Heller MO, Diederichs G, Janz V, Wenzl M, Perka C. Standardized AP radiographs do not provide reliable diagnostic measures for the assessment of acetabular retroversion. $J$ Orthop Res. 2012;30:1369-1376.

29. Weir A, de Vos RJ, Moen M, Hölmich P, Tol JL. Prevalence of radiological signs of femoroacetabular impingement in patients presenting with long-standing adductor-related groin pain. $\mathrm{Br} J$ Sports Med. 2011;45:6-9.

30. Zaltz I, Kelly BT, Hetsroni I, Bedi A. The crossover sign overestimates acetabular retroversion. Clin Orthop Relat Res. 2013;471:2463-2470. 